\title{
Percepción visual y escritura en estudiantes de segundo a cuarto año de e.g.b. del Colegio San José la Salle
}

\section{Visual perception and writing in students from second to fourth year of e.g.b. from the San José la Salle school}

\author{
Francis Ortiz Benalcázar \\ Universidad Central del Ecuador, Ecuador \\ Johanna Bustamante Torres \\ Universidad Central del Ecuador, Ecuador
}

Autor para correspondencia: panchis_michelita92@hotmail.es,jpbustamante@uce.edu.ec Fecha de recepción: 23 de julio de 2018 - Fecha de aceptación: 20 septiembre de 2018

Resumen: El presente artículo basó su investigación en establecer la relación de la percepción visual y la escritura de los niños y niñas de segundo, tercer y cuarto año de Educación General Básica del Colegio San José "La Salle" de la ciudad de Quito en el año lectivo 2016 - 2017. Considerando que la percepción visual permite el reconocimiento cortical, resultante las experiencias sensitivas subjetivas, específicamente de los estímulos que ingresan por vía visual. Por otro lado, la escritura como representación gráfica que posibilita el registro del pensamiento humano y la transmisión óptima de datos e información para relacionarse con el medio donde se desenvuelve la persona. Se sustenta en el enfoque cuantitativo, en la investigación documental, de campo y descriptiva. El marco teórico está debidamente sustentado. Los resultados manifiestan que un gran porcentaje de niños y niñas presentan dificultades en la percepción visual, habilidad pre- requisita básica para el aprendizaje de la escritura, implicando de esta manera la existencia de errores en la misma. Se concluye la ausencia de habilidades de percepción visual; por lo cual es indispensable la intervención por parte de los docentes, para así disminuir posibles dificultades en la escritura en los y las estudiantes.

Palabras claves: percepción visual; escritura; ortografía natural; grafismo; errores específicos

\begin{abstract}
The article based its research on establishing the relationship between the visual perception and the writing skill of children from the second, third and fourth year of General Basic Education of the School San José "La Salle" of Quito city in the school year 2016-2017. By considering that the visual perception allows the cortical recognition, resulting from the subjective sensory experiences. Specifically, of the stimulus that enters by the eyes. On the other hand, the writing is a graphic representation that enables the record of human thought and the optimal transmission of data and information to relate to the medium in which the person develops. It is based on the quantitative approach, on a documentary, field, and descriptive research. The theoretical framework is duly supported. The results manifest that a great percentage of children present difficulties in visual perception, basic pre-requisite skill for learning of writing, thus implying the existence of errors in it. It concludes that absence of skills of visual perception, therefore, intervention by teachers is essential, in order to reduce possible writing difficulties for students.
\end{abstract}

Key Words: visual perception; writing skills; natural spelling; graphic design; specific errors 


\section{Introducción}

Sin duda la escritura hoy en día es uno de los medios de comunicación humana más útil, pues a través de ella se pueden emitir pensamientos, ideas, sentimientos, acciones y dejarlos plasmados en un contenido para la transmisión de datos e información veraz, reflexiva, organizada, técnica e incluso de entretenimiento, permitiendo la adecuada comunicación entre los seres humanos. Además, al ser uno de los medios básicos de comunicación posibilita el aprendizaje, esto la hace convertirse en una destreza importante y muy necesaria en la actualidad; sin embargo, a pesar de ser una de las formas de transferencia de información con tal importancia, no se le da el verdadero valor que deberíamos y nos olvidamos por completo que el aprender a escribir, siendo que el escribir de manera adecuada contribuirá a lograr tanto el desarrollo académico, personal como social de toda persona.

Y aunque, si bien es cierto que el aprendizaje y el desarrollo de la escritura varía de una persona a otra, no se puede dejar de lado que para que se llegue a tener un nivel adecuado de escritura es imprescindible tener ciertos pre-requisitos que sirvan como pilares fundamentales para aprenderla y desarrollarla. El lenguaje oral, la praxia de la escritura, la percepción visual, la orientación, las gnosias en general, etc. son funciones cerebrales que deben estar totalmente dominadas, ya que si no lo están difícilmente un/a niño/a podrá aprender a escribir correctamente.

Con lo dicho anteriormente, se destaca que son algunas las causas existentes para que la escritura pueda verse afectada; sin embargo, esta investigación se centrará en aquella que tiene que ver con los problemas madurativos de la percepción visual. Siendo que, la percepción visual hace referencia a la imagen mental producto del procesamiento de experiencias sensoriales visuales del mundo físico que nos rodea. Dicha percepción permitirá que toda persona pueda reconocer formas geométricas, fisonomías, planos, aprecie distancias, se oriente en el espacio, etc. Todas y cada una de las funciones anteriormente mencionadas resultan imprescindibles al momento de escribir.

Por lo tanto, con todo lo expuesto y tomando en cuenta que un buen nivel madurativo de la percepción visual es esencial para el aprendizaje y desarrollo adecuado de la escritura y por ende de la No aparición de dificultades en la misma, esta investigación se ha planteado determinar la relación de la percepción visual con la escritura de los niños y niñas de segundo, tercero y cuarto de Educación General Básica del Colegio San José "La Salle" con el fin de diseñar una guía metodológica sobre la estimulación y afianzamiento de la percepción visual y la escritura, para que esta manera los profesionales encargados puedan trabajar de mejor manera con los estudiantes.

\section{Desarrollo}

\section{Percepción visual}

Es importante partir recordando que, de todas las modalidades sensoriales, la más estudiada ha sido siempre la visión. Pero en la visión no solo están implicados fenómenos físicos, fisiológicos y neurológicos que producen sensaciones y experiencias visuales, sino también la puesta en marcha de procesos cognitivos superiores, es ahí que cabe exactamente a lo que hace referencia Lescano $(2009$, p.29) que "El cerebro no sólo ha de ver una sola percepción 
de una imagen, sino que ha de entender lo que ve, ha de extraer un significado, recordar y ser capaz de reproducir lo que ha visto".

Otros autores la definen como una actividad integral altamente compleja que involucra el entendimiento de lo que se ve (Koppitz, 1970), y permite organizar y procesar todos los estímulos visuales para así entender el mundo en que vivimos (Martin, 2006). Los autores antes mencionados coinciden en que la percepción visual, primero es un conocimiento y segundo que se obtiene como resultado de estímulos luminosos o visuales. De ahí que la definición más adecuada, tomando en cuenta lo anterior, es que la percepción visual es aquella imagen mental producto del procesamiento de experiencias sensoriales visuales del mundo físico que nos rodea.

Garzia (1996) subdivide a la percepción visual en tres sistemas:

- El sistema visoespacial

- El sistema de análisis visual

- El sistema visomotor

EI sistema visoespacial: hace referencia a la serie de habilidades utilizadas para entender conceptos direccionales que organizan el espacio visual externo, es decir que estas habilidades permiten diferenciar conceptos como: arriba - abajo, adelante - atrás, derecho - izquierdo; conceptos que son esenciales a la hora de desarrollarnos en el medio. Es así que, con estas habilidades el individuo desarrolla la conciencia de su cuerpo con relación al espacio y la relación existente entre los objetos y él. Cuando se hace referencia al sistema visoespacial es importante mencionar que éste se subdivide en tres habilidades:

- La integración bilateral: es habilidad para usar los dos lados del cuerpo en forma simultánea y por separado de una forma consciente, y permite dar el fundamento motor para comprender la diferencia entre los lados derecho e izquierdo del cuerpo.

- La lateralidad: es la habilidad para identificar la derecha e izquierda sobre sí mismo de una forma consciente.

- La direccionalidad: es la habilidad para interpretar direcciones hacia la izquierda o derecha en el espacio exterior. Esta resulta muy importante en el ámbito escolar ya que gracias a ella podemos distinguir conceptos direccionales en la orientación espacial de los símbolos lingüísticos - como b y d-.

El sistema de análisis visual: hace referencia al grupo de habilidades usadas para reconocer, recordar y manipular la información visual, es decir que estas habilidades permitirán observar las diferencias y similitudes entre objetos, formas y símbolos, además de recordarlos y volverlos a visualizar. Este sistema se subdivide en cuatro habilidades:

- Percepción de la forma: permite discriminar y reconocer e identificar formas y objetos. Martin (2006) la divide en cuatro categorías: discriminación visual (habilidad para darse cuenta del tamaño, color y orientación de las formas, y así determinar similitudes o diferencias), figura - fondo (permite atender a un aspecto específico de la forma mientras mantiene consciencia de las relaciones entre la forma y la información del fondo), cierre visual (habilidad para reconocer las claves de un arreglo visual que le permita al individuo determinar la forma final sin necesidad de tener todos los detalles presentes) y constancia de 
forma (habilidad para identificar los aspectos invariantes de la forma cuando se ha alterado el tamaño, la rotación o la orientación).

- Atención visual: proceso de búsqueda de estímulos que influyen en el procesamiento de la información (Berman y Colby, 2009).

La atención visual se compone de tres elementos: llamar la atención (habilidad para centrar la atención en los requerimientos de una tarea específica, en el ámbito escolar le permite al niño involucrarse apropiadamente en la actividad asignada), tomar decisiones (hace referencia al proceso cognitivo de realizar las tareas rápida e inadecuadamente o reflexivamente y resolviendo los problemas lentamente) y mantener la atención (la habilidad para mantener la atención una vez que se ha empezado la tarea) (Garzia, 1996).

- Velocidad perceptual: se refiere a la habilidad para realizar tareas de procesamiento visual de una manera rápida, pero con un esfuerzo cognitivo mínimo, es decir que la información visual captada sea procesada rápida y eficazmente.

- Memoria visual: es la habilidad para recordar el material visualmente presentado. Usualmente se evalúan dos tipos de memoria: memoria espacial (se refiere a la habilidad para recordar dónde está localizado espacialmente un objeto) y memoria secuencial (se refiere a la habilidad para recordar el orden exacto de ítems en una secuencia organizada de izquierda a derecha).

El sistema visomotor: hace referencia a la habilidad general que permite la coordinación de destrezas de procesamiento visual con destrezas motoras. Uno de los componentes de la integración visual-motora es la habilidad para integrar la percepción de la forma con el sistema motor fino para reproducir patrones visuales complejos (Beery y Beery, 2006), es decir que estas dos habilidades se fusionen y puedan trabajar conjuntamente para lograr resultados positivos en la reproducción de patrones. Se requiere de destrezas básicas para reproducir formas complejas como son:

- Percepción visual de la forma, que como se explicó con anterioridad es la que permite discriminar y reconocer e identificar formas y objetos.

- Coordinación motora fina, que es la habilidad para manipular objetos pequeños, por ejemplo: lápices, bolígrafos, etc. (Hammond, 2002)

- Integración de los sistemas visual y motor, que depende de la habilidad para coordinar la percepción interna del espacio con el sistema motor fino para, por ejemplo, copiar letras y números (Beery y Beery, 2006)

\section{Evaluación de la percepción visual}

Para la evaluación de la percepción visual muchos investigadores, educadores y hasta clínicos toman como referencia el estudio por separado de las habilidades de percepción visual específicas ya que hay razones comprensibles para asumir que estas son independientes, sin embargo, a pesar de su estudio en forma aislada no se debe olvidar que de manera práctica se encuentran probablemente entrelazadas de modo inseparable dentro de los individuos.

Para su fácil evaluación varios investigadores han desarrollado pruebas o test para medir habilidades específicas de percepción visual y espacial y programas de intervención específicos para entrenar esas habilidades. Uno de estos es el Test de Figuras Idénticas, con el que se pretendían medir la velocidad perceptual, definida por ellos como la "velocidad en la 
comparación de figuras o símbolos, explorando para encontrar figuras o símbolos o llevando a cabo otras tareas muy simples relacionadas con la percepción visual", el cual se inspira en el de Thurstone en el año 2004.

En Psicología los test sobre rotación, visión y percepción espaciales, se utilizan para medir varios factores relacionados con la inteligencia (Thurstone, 2004), así como de percepción de habilidades viso- espaciales. Dicho test para Thurstone $(2013$, p. 9) "resulta útil en todas las ocasiones en que se desee apreciar las dotes de atención, así como la rapidez y facilidad perceptivo-visual". Para cuantificar la visión espacial existen varias pruebas, una de ellas es el test de Frostig. Este test reúne muchas características por lo que se convierte en uno de los mejores y más utilizados para evaluar habilidades visoespaciales. Este test evalúa coordinación mano - ojo, posición el espacio, copia, figura - fondo, relaciones espaciales, cierre visual, velocidad visomotora y constancia de forma.

\section{Escritura}

Para Pérez (2008, p.1) la escritura es “(...) plasmar pensamientos en un papel u otro soporte material a través de la utilización de signos. Estos signos, por lo general, son letras que forman palabras." Esta definición pone su énfasis, en que la escritura es una manera de comunicar lo que el sujeto piensa o reflexiona sobre algún objeto o acontecimiento, plasmándolo por medio de signos en cualquier material, que luego el lector podrá decodificar y comprender dichos pensamientos e incluso llegar a refutarlos.

Una de las definiciones que también aporta es la que indica que la escritura significados, (2017, p.1). "Es la forma en que fijamos, mediante un conjunto de signos Figuras, el lenguaje con el que hablamos. Es el modo en que los seremos humanos nos comunicamos y transmitimos información, ideas, conceptos, conocimiento o sentimientos de manera no oral (...)." Para este autor, la escritura es el medio de transmisión de ideas de una manera no oral y que permiten expresar nuestros pensamientos, sentimientos, experiencias, entre otros a través de signos gráficos.

Tomando en cuenta las definiciones anteriores y para cumplir con el objetivo de la investigación, se define a la escritura como el sistema que mediante signos convencionales y visibles, permite la materialización de una lengua y la transmisión de la misma. El expresar y comunicar ideas es una necesidad universal e histórica, ya en la antigüedad, el ser humano lo hacía a través de señales, gestos o de manera oral, y tanta fue la necesidad de seguir transmitiendo información que se creó pictogramas, Figuras, símbolos, signos que faciliten su transmisión, hasta llegar a lo que hoy conocemos como la escritura alfabética.

De manera general se puede decir entonces, que la escritura nos permite comunicarnos con nuestros contemporáneos y transmitir nuestros pensamientos a las generaciones futuras, por lo tanto "la escritura es elemento básico de nuestra cultura y garantía de su porvenir" (UNESCO, s.f., p.1), es decir que a través de la escritura todo ser humano puede desenvolverse y desarrollarse de mejor manera en la sociedad. Siendo que, la capacidad para escribir no es sólo el sello de la instrucción elemental, sino que como se dijo anteriormente, es un medio esencial para el progreso individual y el bienestar colectivo. Por tal razón la sociedad se ha interesado por 
saber cuáles son los métodos más eficaces para enseñar a los niños y adultos a escribir en forma legible.

Como manifiesta Gray (2013) “por fortuna muchos de los problemas básicos que entraña la enseñanza de la escritura han sido estudiados por siglos y se ha aprendido bastante acerca de las dificultades de aprender a escribir bien y el valor relativo de los diferentes métodos de la enseñanza de la escritura.". Es así que las investigaciones de años recientes aclaran aún más la naturaleza de la escritura y los factores que influyen en su desarrollo y fortalecimiento durante los primeros años de vida.

\section{Proceso de adquisición y afianzamiento de la escritura}

La escritura como proceso ha sido explicada por los modelos cognitivos contemporáneos de la escritura, estos dan cuenta de las operaciones cognitivas que ocurren cuando los humanos escriben de una manera competente y adecuada. Es así que, antes de consolidar la escritura, es necesario que el niño atraviese ciertas etapas de afianzamiento que le permitirán llegar a la habilidad de escribir. Motivo por el cual, a continuación se explicaran las fases de la consolidación de la escritura desde sus inicios.

\section{Fase pre escritora}

Al inicio del aprendizaje de la escritura el niño o la niña, comienza a desarrollar la conciencia fonológica, pero aún no es capaz de reflexionar sobre la estrecha relación entre los signos gráficos y los sonidos de las letras y/o números, por lo que su escritura se basa en la reproducción de dibujos (véase figura 1). En esta etapa la descarga motriz a través del movimiento del lapicero sobre el papel es lo más significativo, siendo cierto que los niños que se encuentran en esta edad abordan los tres años de vida y es ahí donde aparecen los primeros grafismos (garabatos o trazos sin sentido), estos durante esta instancia tiene solamente intenciones lúdicas.

Otra característica de esta etapa es el ambidextrismo, que es el uso simultáneo de ambas manos y por ende de los dos hemisferios cerebrales. El grafismo de los niños y las niñas que se encuentran en esta etapa no tiene control de frenado, ya que el grafismo aquí es impulsivo. Ya pasados los tres primeros años el niño ya inicia la representación intuitiva, es decir va cediendo la impulsividad motriz, mejora el soporte del lapicero y le da una significación a lo que dibuja. Es ya a partir del cuarto año que el niño inicia el proceso grafomotor, el mismo que le permite reproducir formas geométricas sencillas. Aquí la lateralidad ya se está definiendo por lo que el cambio de mano es infrecuente. Inicia así, el dominio del espacio gráfico.

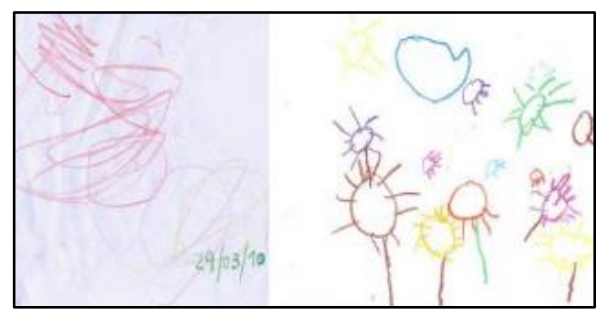

Figura 1. Fase Pre escritora - Fuente: Triones y Gallardo (2017). Psicología del desarrollo: La evolución del gesto figura y el desarrollo de la grafomotricidad 


\section{Fase escritora}

Para esta fase los niños ya han cumplido los cinco años y aquí es donde inicia el aprendizaje sistemático de la escritura. Durante esta etapa los niños comienzan a asociar los fonemas con los grafemas, el niño ya empieza a copiar letras aisladas y palabras cortas. En esta fase por lo general los niños y niñas cometen un sinnúmero de errores de la escritura (sustituciones, escritura en espejo, omisiones, adiciones, entre otros). Siendo que a los seis años los trazos aún son un tanto temblorosos y la inclinación y dimensión de la letra aun no es controlada en su totalidad. Conforme pasen los años, el niño va afianzando hasta adquirir la destreza de escribir y lograr almacenar un patrón ortográfico de numerosas palabras en su almacén léxico ortográfico que le permitirá dejar de cometer errores en la escritura. Por ello resulta necesario como manifiesta Rosas (2012) "No se debe forzar los procesos de maduración sino al contrario debemos respetar la integración neuromotriz para evitar el fracaso del escolar".

\section{Etapas del desarrollo de la escritura}

Una vez automatizada la escritura como técnica instrumental básica, el niño desplazará su atención a ciertos otros aspectos de esta como la ortografía, gramática, sintaxis y el contenido que quiere comunicar. Para esto es igual de importante que atraviese ciertas etapas.

\section{a) Etapa pre caligráfica}

Esta etapa se desarrolla de los 6 a 9 años aproximadamente. Abarca todo el período de adquisición de las destrezas gráficas especializadas, desde que el garabato intenta ser una letra, hasta el dominio de la caligrafía. En esta etapa según Condemarín y Chadwin (1991) se observan los siguientes rasgos: "Trazos rectos de las letras temblorosos o curvos, Curvas o semicurvas de las letras que presentan ángulos, Dimensión e inclinación de letras no regulares, Ligado entre letras, Alineación no recta, tiende a ser ondulante"

Los niños y niñas que al no lograr superar esta etapa pre caligráfica, al llegar a los no alcanzar a los 9 años no afianzarán la escritura y desarrollaran disgrafía con características propias, tales como: simetría inadecuada, presencia elevada de ángulos, dimensión exagerada de la letra, inclinación a la izquierda o recta, unión y soldadura de palabras, inadecuado espacio entrelineado.

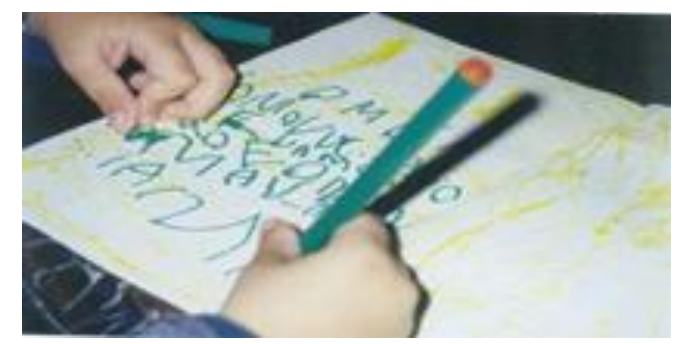

Figura 2. Fase pre caligráfica

Fuente: Triones \& Gallardo (2017). Psicología del desarrollo: la escritura, etapa pre caligráfica.

\section{Etapa caligráfica}


Esta etapa se desarrolla de los 10 a 12 años aproximadamente, ya que se inicia cuando el niño ha manifestado un dominio de su motricidad fina. Su escritura corresponde a la ideal caligrafía.

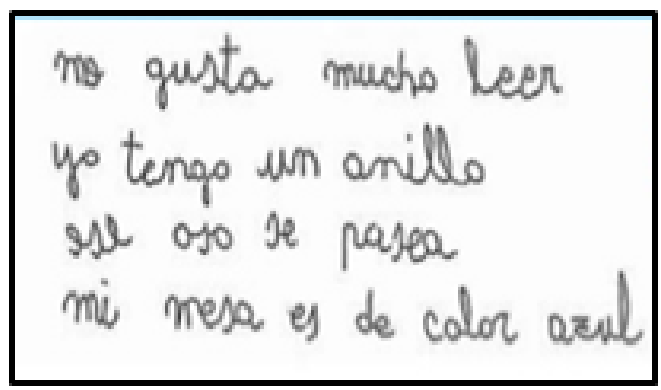

Figura 3. Etapa caligráfica

Fuente: Miranda (2014). Escritura: desarrollo y evaluación.

\section{Etapa post caligráfica}

Esta etapa se desarrolla y fortalece desde los 12 años en adelante. Aquí se observa una tendencia a realizar caligrafía ya personalizada, mientras que la rapidez en la escritura juega un rol muy importante en esta etapa.

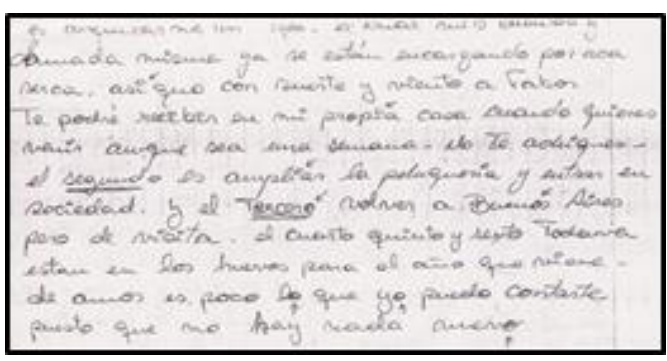

Figura 4. Etapa post caligráfica

Fuente: Miranda (2014). Escritura: desarrollo y evaluación

\section{Manifestaciones de la escritura}

Para que el niño o la niña inicie el proceso escritor, debe cumplir ciertas normas básicas que le facilitarán su aprendizaje, tales como: sentarse en un ángulo de 90 grados, ubicando su cuerpo en L frente a la mesa, controlar la sujeción del lápiz, al moverlo y detenerlo en su movimiento por el papel. Una vez instauradas estas conductas el niño estará en plena capacidad para desempeñarse en la escritura en todas sus manifestaciones de manera correcta.

Copia: Al hablar de copiar se hace referencia al acto de reproducir o imitar ciertas conductas manuales que dan lugar a determinados estímulos visuales. Para lograr esto el niño debió haber adquirido ciertas conductas motrices manuales básicas que suelen desarrollarse a través de la reproducción de modelos gráficos. En tal sentido, la copia permite adquirir conocimiento acerca de si el niño puede leer lo que copió y la calidad del grafismo con la que lo hace, además permite observar el paralelismo de los renglones, la dirección del trazo de las letras y la mano que utiliza para escribir (lateralidad). 
Dictado: La escritura al dictado es un proceso más complejo que la copia, ya que requiere tener a la retentiva auditiva en un buen nivel y, al mismo tiempo, haber interiorizado previamente los grafemas y su correspondiente relación fonemática, así como la secuenciación u ordenación de los estímulos auditivos que a posterior se transformarán en lenguaje escrito. Es así que, el dictado involucra la correspondencia existente en un código o idioma dado entre fonemas y grafemas, es decir, que los estímulos auditivos expresados por la persona que dicta deben ser descompuestos en sus elementos. Mediante el dictado podremos observar si el niño ha adquirido la capacidad de discriminar los fonemas, los mismos que deben ser retenidos secuencialmente para luego ser trazados.

Escritura espontánea: Al referirnos a escritura espontánea hacemos alusión a la expresión de ideas propias a través de la escritura. Sin duda de las manifestaciones de la escritura, esta es la de mayor complejidad, puesto que al no estar presente el modelo visual o auditivo a reproducir es necesario que exista un buen lenguaje interior en el niño. La escritura espontánea, llamada composición escrita, es la máxima aspiración en el aprendizaje de la escritura, aunque es el proceso que más tarda en automatizarse.

\section{Parámetros de evaluación de la escritura}

La evaluación de la escritura es importante para poder identificar las dificultades que el niño o la niña presentan durante su proceso de afianzamiento, e incluso, a partir de los 9 años es indispensable que cuando se detecten ciertas irregularidades en el proceso de aprendizaje, la escritura debe ser evaluada con la finalidad de dar una presunción diagnóstica e intervenir a tiempo. Para la evaluación de la escritura existen tres aspectos esenciales en los cuales se debe basar: grafomotricidad, composición y ortografía.

Grafomotricidad: Al hablar de grafomotricidad se hace referencia a la capacidad del ser humano para ejecutar con precisión y eficacia la producción física de determinados modelos gráficos a través de movimientos, habilidades y destrezas. Dentro de esta temática se han recolectado de varios autores los siguientes indicadores a observar, los cuales serán de suma importancia para la futura evaluación:

- Tamaño de la letra: se presenta en la uniformidad de las letras, por lo tanto, el error va a estar contemplado en la igualdad de mayúsculas y minúsculas, ya que la primera debe tener una altura mayor que las demás.

- Regularidad: es cuando la escritura presenta irregularidades en la altura, anchura, inclinación y dirección de lo escrito.

- Oscilación: el error se basa cuando la escritura presenta trazos temblorosos.

- Líneas anómalas: dentro de la escritura de una palabra se hallan líneas fragmentadas u onduladas.

- Interlineado: error correspondiente al no respetar el espacio entre líneas altas, media y baja en la escritura.

- Distribución de zonas: el error se comete al visualizar en una palabra una letra que no respeta el espacio del anterior o siguiente grafema.

- Soldaduras: el error se logra visualizar cuando una palabra es unida con otra. 
- Calidad de curvas: se manifiesta en la prensión que se produce al momento de trazar con el lápiz sobre el papel. El exceso de prensión del lápiz produce una peor calidad de curvas.

- Calidad de líneas verticales: escribir erróneamente las líneas verticales que presentan algunos grafemas.

Ortografía: Para Moena y Orellana (2012, p.5). "La ortografía hace referencia al conjunto de habilidades que nos permiten codificar gráficamente de manera apropiada la palabra hablada”. Es decir, escribir correctamente por el correcto empleo de letras y de los signos de la escritura. Entre los indicadores de evaluación en esta área y los posibles errores que se pueden presentar encontramos:

- Sustitución de letras: error que se presenta al momento de reemplazar una letra por otra (ejemplo: lima por cima).

- Adiciones: error que consiste en añadir un grafema no correspondiente a la palabra escrita. Puede producirse debido a un problema de concentración, de memoria operativa o por no tener almacenada una imagen mental de la palabra. (ejemplo: marrtillo por martillo).

- Omisiones: al momento de escribir se omite, una letra, sílaba o palabra. (honestidad por onestidad)

- Inversión de orden: se produce un error al cambiar el orden de una palabra dentro de una oración o de un grafema dentro de una palabra. Esto se debe a que no existe comprensión de la palabra u oración y solo se concentra en la decodificación de estas, sin tener un orden. (ejemplo: saca por casa).

- Uniones de palabras: el error se comete, principalmente, originado por una percepción auditiva deficiente. Escucha la oración como un todo, sin estimar en la separación de palabras (ejemplo: lacasa por la casa).

- Fragmentaciones: el error se presenta en la separación o segmentación de una palabra, generalmente, en dos partes. Esto se origina debido a que no se tiene una imagen mental de la palabra (ejemplo: arma rio por armario).

Composición escrita: Al hablar de composición escrita, se hace referencia a la utilización de la escritura como instrumento de expresión de ideas, sentimientos, pensamientos, etc. Según Moena y Orellana (2014) afirman que "la composición es a la escritura, lo que la comprensión es a la lectura", es decir que la composición juega un papel muy importante en el desarrollo de la escritura y por ende en el desenvolvimiento del individuo dentro de la sociedad.

Estas composiciones deben ser textos estructuralmente apropiados, con construcciones sintácticas correctas, con vocabulario precios y amplio, y utilizando con propiedad los signos de puntuación. Para Moena y Orellana (2014) los indicadores que permiten observar la existencia de algún error en la composición escrita son: a) Adecuación y riqueza léxica, b) construcciones gramaticales, c) organización del texto, las mismas que se caracterizan a continuación:

- Adecuación y riqueza léxica: se refiere a hacer uso de un vocabulario variado, complejo y apropiado al tema.

- Construcciones gramaticales: es la adecuación de las estructuras sintácticas empleadas, donde está presente el uso de los tiempos verbales, pronombres, artículos, las preposiciones, etc. 
- Organización del texto: dentro de este se toma en cuenta el título (que debe expresar en pocas palabras el tema que trata la información. Debe despertar el interés del lector), la introducción (en ella se da a conocer el tema del texto, se expone el propósito del autor), el desarrollo (se desarrolla el tema a través de ideas principales y secundarias), la conclusión (breve síntesis de lo expuesto. Se recapitula lo más relevante del tema tratado) y formato y presentación (se refiere a que la presentación debe ser ordenada, con letra legible y un vocabulario acorde a la edad).

\section{Metodología}

Para la medición de las variables que se estudiaron, se determinó la utilización de la técnica psicométrica, ya que esta permitirá seleccionar el instrumento más adecuado y pertinente. Así también se utilizará la observación directa ya que se entrará en contacto con el objeto de estudio que en este caso son los niños y niñas de segundo, tercer y cuarto año de E.G.B.

Para la variable independiente "La percepción visual" se utilizó la técnica psicométrica y como instrumento especifico el test DTVP-2 de Desarrollo de la Percepción Visual de FROSTIG, el cual ha sido diseñado con el propósito de apreciar los retrasos en la madurez perceptiva en niños que presentan dificultades de aprendizaje, con edades comprendidas entre los 3 y los 7 años de edad. El DTVP-2 es una adaptación del test original de Frostig, es una edición actualizada que explora ocho aspectos de la percepción visual que son relativamente independientes: coordinación ojo-mano, posición en el espacio, copia, figura - fondo, relaciones espaciales, cierre visual, velocidad visomotora y constancia de forma.

Para la variable dependiente "La escritura" de igual manera se utilizará la técnica psicométrica y como instrumento específico el subtest de escritura del Test de Análisis Lecto Escritor T.A.L.E, el cual tiene como objetivo la evaluación de los niveles generales y las características específica de la escritura de cualquier niño en un momento dado del proceso de adquisición de tales conductas.

\section{Resultados Y Discusión}

Los instrumentos utilizados reúnen las condiciones de confiabilidad y validez como características fundamentales para que se garanticen la idoneidad de los datos y los resultados. Es así, que luego de la aplicación de los test, se procedió a la tabulación de cada uno sacando puntuaciones de cada estudiante en las áreas percepción visual y escritura. Sus resultados fueron cuantificados según el baremo del instrumento psicométrico y luego transformados a porcentajes, lo que facilitó el trabajo de análisis mediante cuadros y gráficos que fueron procesados en Excel. 
Tabla 1. Resultados de habilidades viso-espaciales de los niños y niñas de $2^{\circ}, 3^{\circ}$ y $4^{\circ}$ año de $E G B$

\begin{tabular}{|c|c|c|c|c|c|c|}
\hline $\mathrm{N}^{\circ}$ & Subpruebas & 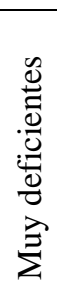 & 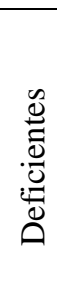 & 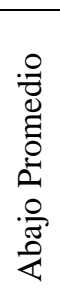 & $\begin{array}{l}\stackrel{O}{0} \\
\overparen{D} \\
0 \\
0\end{array}$ & 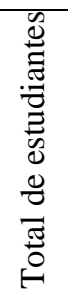 \\
\hline 1 & Coordinación ojo-mano & 2 & 9 & 30 & 139 & 180 \\
\hline 2 & Posición en el espacio & 5 & 22 & 36 & 124 & 187 \\
\hline 3 & Copia & 0 & 0 & 1 & 27 & 28 \\
\hline 4 & Figura -fondo & 6 & 39 & 49 & 89 & 183 \\
\hline 5 & Relaciones espaciales & 0 & 0 & 6 & 31 & 37 \\
\hline 6 & Cierre Visual & 37 & 50 & 50 & 53 & 190 \\
\hline 7 & Velocidad Visomotora & 0 & 0 & 0 & 29 & 29 \\
\hline 8 & Constancia de forma & 1 & 14 & 59 & 122 & 196 \\
\hline
\end{tabular}

Fuente: Aplicación del test de FROSTIG

La tabla 1 indica la cantidad de niños y niñas en las escalas desde promedio hasta muy deficiente de $\operatorname{los} 2^{\circ}, 3^{\circ}$ y $4^{\circ}$ años de EGB, para luego relacionar las habilidades viso-espaciales no fijadas y poder establecer el grupo de intervención tomando en cuenta a los grupos que han presentado mayor dificultad: deficientes y muy deficientes.

Tabla 21. Escala para medir los niveles de errores por curso

\begin{tabular}{ccccc}
\hline Curso & Promedio & \multicolumn{3}{c}{ NIVELES DE ERRORES } \\
& & Máximo & Medio & Mínimo \\
\hline 2do "A" & $0-8$ & $6-8$ & $3-5$ & $0-2$ \\
2do "B" & $0-29$ & $20-29$ & $10-19$ & $0-9$ \\
3ro "A" & $0-24$ & $17-24$ & $9-16$ & $0-8$ \\
3ro "B" & $0-10$ & $8-10$ & $4-7$ & $0-3$ \\
4to "A" & $0-12$ & $8-12$ & $4-7$ & $0-3$ \\
4to "B" & $0-49$ & $34-49$ & $17-33$ & $0-16$ \\
\hline \multicolumn{4}{c}{ Fuente: Aplicación del test de T.A.L.E. }
\end{tabular}

En base al nivel de errores se toma en cuenta:

1. Nivel Máximo: Errores graves. No afianzamiento de la escritura. Inmediata intervención

2. Nivel Medio: Errores moderados. Grado de dificultad medio en la escritura. Necesita Intervención.

3. Nivel Mínimo: Errores leves. Grado de dificultad menor en la escritura o suficiencia en la escritura. Ninguna Intervención

4. Se realiza un promedio (mismo número de intervalos) 
Tabla 3. Escala para medir los niveles de errores institucionalmente

\begin{tabular}{ccccc}
\hline Cursos & Promedio & Máximo & Medio & Mínimo \\
\hline 2do a 4to & $0-49$ & $33-49$ & $17-32$ & $0-16$ \\
\hline \multicolumn{4}{c}{ Fuente: Aplicación del test de } & T.A.L.E.
\end{tabular}

Tabla 4. Resultados finales de errores de ortografía natural

Clasificación Descriptiva (Nivel) Número de estudiantes $\quad$ Porcentaje

\begin{tabular}{lcc}
\hline Máximo & 8 & 3,83 \\
Medio & 40 & 19,14 \\
Mínimo & 161 & 77,03 \\
TOTAL & 209 & 100,00 \\
\hline
\end{tabular}

Fuente: Aplicación del test de T.A.L.E., sub prueba Ortografía Natural

De acuerdo al registro de errores en la ortografía natural, 161 estudiantes que corresponde al 77,03\% demuestran que tienen errores leves o grado de dificultad menor en la ortografía natural; 40 estudiantes que corresponde al 19,14\% presentan errores moderados o grado de dificultad medio en la ortografía natural y 8 estudiantes que corresponde al 3,83\% demuestran errores graves o no afianzamiento de ortografía natural de la escritura. Por lo tanto, la mayoría de estudiantes investigados poseen la suficiencia en la ortografía natural de la escritura al no presentar mayores dificultades.

Tabla 5. Ficha general de resultados de errores de ortografía natural

\begin{tabular}{|c|c|c|c|c|c|c|c|c|c|}
\hline $\mathrm{N}^{\circ}$ & CURSO & 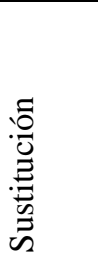 & 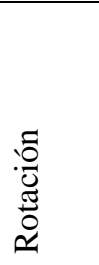 & 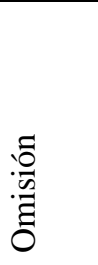 & 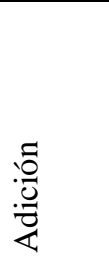 & 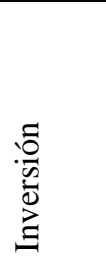 & 总 & 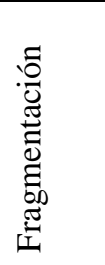 & 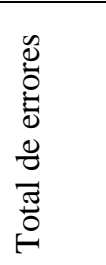 \\
\hline 1 & $2^{\circ} \mathrm{EBG} " \mathrm{~A} "$ & 67 & 0 & 70 & 10 & 3 & 19 & 13 & 182 \\
\hline 2 & $2^{\circ} \mathrm{EBG} " \mathrm{~B} "$ & 78 & 0 & 129 & 19 & 4 & 73 & 18 & 321 \\
\hline 3 & $3^{\circ} \mathrm{EBG} " \mathrm{~A} "$ & 170 & 1 & 152 & 20 & 8 & 3 & 13 & 367 \\
\hline 4 & $3^{\circ} \mathrm{EBG}$ "B" & 151 & 0 & 138 & 7 & 13 & 7 & 9 & 325 \\
\hline 5 & $4^{\circ} \mathrm{EBG} " \mathrm{~A} "$ & 178 & 4 & 210 & 39 & 0 & 9 & 7 & 447 \\
\hline 6 & $4^{\circ} \mathrm{EBG}$ "B" & 145 & 0 & 164 & 18 & 0 & 1 & 7 & 335 \\
\hline \multicolumn{2}{|c|}{ Cantidad de errores } & 789 & 5 & 863 & 113 & 28 & 112 & 67 & 1977 \\
\hline \multicolumn{2}{|c|}{ Porcentaje } & 39,9 & 0,25 & 43,7 & 5,72 & 1,42 & 5,67 & 3,39 & 100 \\
\hline
\end{tabular}

Fuente: Aplicación del test de T.A.L.E.

Según el resumen de los errores de ortografía natural cometidos por los 209 estudiantes de segundo, tercer y cuarto año EGB, se encontraron en el momento del dictado y la copia, la investigación los siguientes resultados : 863 omisiones que corresponde 43,7\%, 789 sustituciones que corresponde al 39,9\%, 113 adiciones que corresponde al 5,72\%, 112 uniones que corresponde al 5,67\%, 67 fragmentaciones que corresponde al 3,39\%, 28 inversiones que 
corresponde al $1,42 \%$ y 5 rotaciones que corresponde al $0,25 \%$. Por lo tanto, se puede deducir que los errores más comunes cometidos por los estudiantes en ortografía natural son omisiones, sustituciones, adiciones y uniones, y en cambio con porcentajes menores se cometen los errores de fragmentaciones, inversiones, y rotaciones.

Tabla 6. Escala para medir los niveles de errores por curso

\begin{tabular}{ccccc}
\hline Curso & Promedio & \multicolumn{2}{c}{ NIVELES DE ERRORES } \\
& & Máximo & Medio & Mínimo \\
\hline 2do "A" & $0-8$ & $6-8$ & $3-5$ & $0-2$ \\
2do "B" & $0-29$ & $20-29$ & $10-19$ & $0-9$ \\
3ro "A" & $0-24$ & $17-24$ & $9-16$ & $0-8$ \\
3ro "B" & $0-10$ & $8-10$ & $4-7$ & $0-3$ \\
4to "A" & $0-12$ & $8-12$ & $4-7$ & $0-3$ \\
4to "B" & $0-49$ & $34-49$ & $17-33$ & $0-16$ \\
\hline
\end{tabular}

Fuente: Aplicación del test de T.A.L.E.

En base al nivel de errores se toma en cuenta:

1. Nivel Máximo: Errores graves. No afianzamiento de la escritura. Inmediata intervención

2. Nivel Medio: errores moderados. Grado de dificultad medio en la escritura. Necesita Intervención.

3. Nivel Mínimo: errores leves. Grado de dificultad menor en la escritura o suficiencia en la escritura. Ninguna Intervención

4. Se realiza un promedio (mismo número de intervalos)

Tabla 7. Escala para medir los niveles de errores institucionalmente

\begin{tabular}{ccccc}
\hline Cursos & Promedio & Máximo & Medio & Mínimo \\
\hline 2do a 4to & $0-49$ & $33-49$ & $17-32$ & $0-16$ \\
\hline \multicolumn{5}{c}{ Fuente: Aplicación del test de } \\
T.A.L.E.
\end{tabular}

Tabla 8. Resultados finales de errores en el grafismo

\begin{tabular}{lcc}
\hline Clasificación Descriptiva & Número de estudiantes & Porcentaje \\
& & \\
\hline Máximo & 0 & 0,00 \\
Medio & 5 & 2,39 \\
Mínimo & 204 & 97,61 \\
TOTAL & 209 & 100,00 \\
\hline
\end{tabular}

Fuente: Aplicación del test de T.A.L.E., sub prueba Grafismo

De acuerdo al registro de errores en grafismo, 204 estudiantes que corresponde al 97,61\% demuestran que tienen errores leves o grado de dificultad menor en la ortografía natural; 5 estudiantes que corresponde al 2,39\% presentan errores moderados o grado de dificultad medio en la ortografía natural y 0 estudiantes que corresponde al 0,00\% demuestran errores graves o no afianzamiento del grafismo de la escritura. Por lo tanto, la mayoría de estudiantes investigados poseen la suficiencia en grafismo de la escritura al no presentar mayores dificultades. 
Tabla 92. Ficha general de resultados de errores en el grafismo

\begin{tabular}{|c|c|c|c|c|c|c|c|c|c|c|c|}
\hline $\mathrm{N}^{\circ}$ & CURSO & 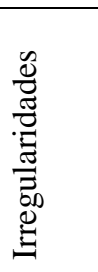 & 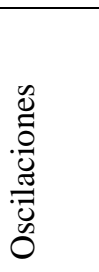 & 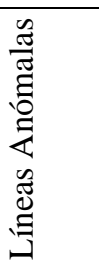 & $\begin{array}{l}\stackrel{\mathscr{\Xi}}{\Xi} \\
\stackrel{\Xi}{\Xi} \\
\stackrel{\Xi}{\Xi}\end{array}$ & 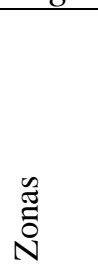 & 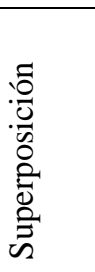 & $\begin{array}{l}0 \\
\frac{\pi}{3} \\
\frac{0}{0} \\
\frac{\sigma}{0} \\
\stackrel{0}{0}\end{array}$ & 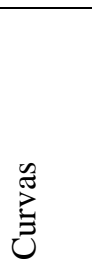 & 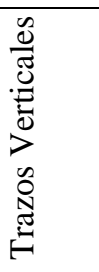 & 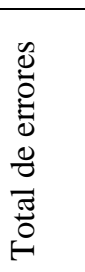 \\
\hline 1 & $2^{\circ} \mathrm{EBG} " \mathrm{~A} "$ & 12 & 0 & 7 & 20 & 15 & 1 & 1 & 5 & 1 & 62 \\
\hline 2 & $2^{\circ} \mathrm{EBG} " \mathrm{~B} "$ & 12 & 1 & 5 & 21 & 23 & 0 & 0 & 2 & 11 & 75 \\
\hline 3 & $3^{\circ} \mathrm{EBG}$ "A" & 26 & 13 & 22 & 20 & 19 & 1 & 1 & 6 & 31 & 139 \\
\hline 4 & $3^{\circ} \mathrm{EBG}$ "B" & 26 & 23 & 25 & 31 & 16 & 2 & 0 & 22 & 37 & 182 \\
\hline 5 & $4^{\circ} \mathrm{EBG} " \mathrm{~A} "$ & 25 & 14 & 22 & 29 & 24 & 11 & 4 & 19 & 20 & 168 \\
\hline 6 & $4^{\circ} \mathrm{EBG} " \mathrm{~B} "$ & 28 & 14 & 20 & 34 & 17 & 2 & 0 & 10 & 30 & 155 \\
\hline \multicolumn{2}{|c|}{ Cantidad de errores } & 129 & 65 & 101 & 155 & 114 & 17 & 6 & 64 & 130 & 781 \\
\hline \multicolumn{2}{|c|}{ Porcentaje } & 16,5 & 8,32 & 12,9 & 19,8 & 14,6 & 2,18 & 0,77 & 8,19 & 16,6 & 100 \\
\hline
\end{tabular}

Fuente: Aplicación del test de T.A.L.E., sub prueba Grafismo

La tabla 9 permite observar el resumen de los errores en el grafismo cometidos por los 209 estudiantes de segundo, tercer y cuarto año EGB, encontrándose en el momento del escritura espontánea, la investigación arrojó los siguientes resultados : 155 errores en las interlíneas que corresponden al 19,8\%; 130 errores en los trazos verticales que corresponde al $16,6 \% ; 129$ irregularidades que corresponde al 16,5\%; 114 errores en las zonas que corresponde al 14,6\%; 101 líneas anómalas que corresponde al 12,9\%; 65 oscilaciones que corresponde al 8,32\%; 64 errores en las curvas que corresponde al 8,19\%; 17 superposiciones que corresponde al $2,18 \%$ y 6 soladuras que corresponde al $0,77 \%$. Por lo tanto, se puede deducir que los errores más comunes cometidos por los estudiantes en el grafismo son interlíneas, trazos verticales, irregularidades, zonas, líneas anómalas, y en cambio con porcentajes menores se cometen los errores de oscilaciones, curvas, superposiciones, y soldaduras.

En cuanto a la primera variable de percepción visual, la mayoría de estudiantes investigados poseen la habilidad para dibujar líneas rectas o curvas con precisión, demostrando la habilidad específica de coordinación viso motriz, y tomando en cuenta que este tipo de coordinación es parte de las conductas perceptivas motrices de base; las cuales, inciden de manera significativa en el aprendizaje como lo explican en la conclusión de su investigación Vilatuña, Guajala y otros (2012, p 142) “Las imágenes, sonidos, sensaciones somáticas, olores, sabores, etc., contribuyen al desarrollo de las formas cognitivas superiores, es decir, que tanto sensación y percepción son fundamentales en la construcción del conocimiento". Por todo ello, es prioritario por parte de los docentes planificar actividades para desarrollar habilidades de coordinación de músculos finos durante las primeras etapas evolutivas, permitiendo en los discentes ejecutar acciones que involucran el ojo y la mano. 
Según Bustamante (2016, p. 79) la percepción viso - motriz o grafo percepción, "incide en el sujeto al integrar la interpretación visual y la coordinación motora gráfica", permitiendo que una persona pueda copiar gráficos o textos; en el caso de la población investigada, la gran mayoría demuestran una habilidad específica para reproducir mediante copia en un nivel superior del promedio, es decir poseen la habilidad para reconocer los rasgos de un diseño y dibujarlos a partir de un modelo, a lo que Beery y Beery (2006) lo nominan como integración de los sistemas visual y motor, ya que permite la percepción interna del espacio con el sistema motor fino. Por otro lado, concibiendo a la orientación espacial como una función cognitiva responsable de la percepción y la ubicación de estímulos en el espacio, es evidente su importancia en la reproducción adecuada de gráficos, influyendo así, en el desarrollo y afianzamiento de lectura escritura y cálculo, como lo manifiesta Cabezas (2014) en las conclusiones de su tesis "la orientación espacial durante el primer año de educación general básica influye directamente en los procesos mentales y en los procesos de aprendizaje (...)". En el mismo sentido, en cuanto a la habilidad figura - fondo se demuestra un adecuado desarrollo en los estudiantes investigados, ya que estos pueden observar figuras específicas cuando están ocultas por un fondo confuso y complejo, permitiendo fortalecer la diferenciación, detección y selección de estímulos visuales dentro de un entorno determinado, y así poder discriminar las formas de manera independiente.

Como lo expone Martin (2006) "permite atender a un aspecto específico de la forma mientras mantiene consciencia de las relaciones entre la forma y la información del fondo". En sentido funcional, la percepción figura-fondo se la debe concebir desde un sentido evolutivo del individuo, ya que permite observar y discriminar cuerpos o figuras tridimensionales de manera precisa. Al mismo tiempo, la velocidad visomotora, todos los estudiantes han logrado la rapidez con la que un niño o niña pueda reproducir señales específicas de acuerdo al desarrollo escritor de su nivel escolar en la que se encuentran. La dificultad se la encuentra en la habilidad de cierre visual, aunque Martin (2006) manifiesta que el cierre visual es la "habilidad para reconocer las claves de un arreglo visual que le permita al individuo determinar la forma final sin necesidad de tener todos los detalles presentes", es notoria la deficiencia de los estudiantes en cuando al desarrollo de esta habilidad, incidiendo en la dificultad para reconocer una figura estímulo que ha sido dibujada de manera incompleta. Es necesario acotar que la visión permite reconocer, discriminar e interpretar las formas e imágenes del mundo exterior, permitiendo incluso completar o cerrar las imágenes que se encuentran segmentadas o no estructuradas. Por lo dicho, los estudiantes que no tienen estructurado el cierre visual, podrían presentar errores en la escritura e incluso en la lectura.

En cuanto a la variable escritura, conocida por permitir la representación gráfica de lo que pensamos o sentimos, como lo manifiesta Pérez $(2008$, p.1) la escritura es “(...) plasmar pensamientos en un papel u otro soporte material a través de la utilización de signos. Estos signos, por lo general, son letras que forman palabras." En este caso, la mayoría de estudiantes alcanzaron un adecuado nivel para su edad en ortografía natural, siendo que los errores más comunes de toda la población investigada fueron: omisión, sustitución, adición, malas uniones, fragmentaciones, inversiones y rotaciones. En cuanto a la estructura gráfica, conocida como grafismo, la mayoría de estudiantes investigados poseen la suficiencia en grafismo de la escritura al no presentar mayores dificultades, ya que su letra se presenta simétrica en la forma y dimensión. Aunque la dificultades son evidentes en de manera común en interlíneas, trazos verticales, irregularidades, zonas, líneas anómalas, en porcentajes menores se cometen los 
errores de oscilaciones, curvas, superposiciones, y soldaduras. La importancia de una adecuada escritura radica en que, como lo dice la UNESCO "la escritura es un elemento básico de nuestra cultura y garantía de su porvenir"; en tal sentido, desde sus inicios el ser humano ha podido pasar información por varias generaciones a través de este mecanismo de comunicación, que incluso no solo transmite sino también que logra adentrarnos en historias o experiencias de otras personas, motivo por el cual los docentes de nivel inicial deben conocer sobre las estrategias metodológicas que incidirán en el desarrollo y potenciación de la escritura en los niños y niñas durante el inicio de su formación académica, con la finalidad de evitar dificultades específicas de aprendizaje, siendo esta muy comunes: la disgrafía y la disortografía.

\section{Conclusiones}

Los resultados de la investigación indican que los niños y niñas de segundo, tercero y cuarto año de E.G.B. del Colegio San José "La Salle" presentan como errores más comunes de Ortografía Natural: omisiones, sustituciones, adiciones y uniones manifestados en la copia y en el dictado; y en cuanto al Grafismo errores como: interlíneas, trazos verticales irregularidades, zonas y líneas anómalas. Lo que indica la ausencia de las conductas de habilidades previas conocidas como requisitos, las cuales abarcan actividades de discriminación visual para su fijación y afianzamiento.

A su vez, los estudiantes del Nivel Máximo, relacionados con errores graves de escritura emitido por el Test T.A.L.E., presentan falta de afianzamiento de la escritura, ocasionada por la ausencia de estímulos discriminatorios adecuados y planificados en los procesos de enseñanza y aprendizaje, por lo cual se requiere de un plan de intervención para afianzar las habilidades de Percepción Visual, tales como posición en el espacio, figura- fondo y cierre visual, mediante una guía de desarrollo de las mismas. Es imperante considerar que las habilidades previas o más simples darán inicio a otras más complejas en los procesos de enseñanza y aprendizaje de la escritura, cuando esas habilidades básicas no han sido afianzadas, el inicio del aprendizaje de la escritura no será satisfactorio.

\section{Bibliografía}

Alberich, J., Ferrer, A., \& Gómez, D. (s.f). La percepción visual. Universidad Oberta de Catalunya. Cataluña - España

Ardila, A \& Roselli, M. (2010). Neuropsicología clínica. Mexico, D.F: Editorial Manual

Moderno Bustamante J. y Andrade X. (2016). Funciones básica, Técnicas activas para su desarrollo y Neuropsicología. Ecuador-Ecuafuturo.

Casado, V. (2016). El sistema nervioso humano: estructuras y funciones. Lifeder.com

Codigo de la niñez y adolescencia. (2003)

Constitución de la República del Ecuador. (2008)

Espinoza, I. (2003). Problemas de Aprendizaje. Quito: Miraflores

Fernández, A. \& Gairin, J. Didáctica de la escritura. Barcelona: Editorial Humanitas. 
García, M. (s.f). Percepción, atención y memoria: Introducción al estudio de la percepción. Madrid - España.

Gray, W. (s.f). La enseñanza de la lectura y de la escritura. ONU para la educación, la ciencia y la cultura. Paris: talleres Atar.

Groffman, S. (2006). The Relationship Between Visual Perceptual Problems and Learning. Optometric Management of Learning-Related Vision Problems (pp. 241-280). Philadelphia: Evolve.

Jurado, F. \& Bustamante, G. (1997) Los procesos de la escritura: Hacia la producción interactiva de los sentidos. Bogotá: Magisterio.

Lescano, P. (2013). La percepción visual en el desarrollo de los procesos cognitivos. Ambato Ecuador

López, M. (2016). Disgrafía y disortografía: diagnóstico y tratamiento en alumnos de 2 E.P.

Merchán, M. (2011). Influencia de la percepción visual en el aprendizaje. Revista de Ciencia, tecnología y salud, volumen 9, pp. 93 -101

Ministerio de Educación (2008). Resultados pruebas censales Ser Ecuador 2008. Ecuador: Bicentenario.

Moena, C. \& Orellana, S. (2012). Qué evaluar en escritura y lectura. Taller de elaboración de instrumentos. Inacap Rancagua Psicopedagogia

Moreano, S. (2015). Las gnosias visoespaciales y las dificultades grafemáticas en la escritura. (Tesis de maestría). Universidad Central del Ecuador, Quito - Ecuador.

Montealegre, R. (2006). Desarrollo de la lectoescritura: adquisición y dominio. Acta Colombiana de Psicología, volumen (9), pp.25-40. Universidad Católica de Colombia, Colombia.

OCDE (s.f). El programa PISA de la OCDE: qué es y para qué sirve. París: OCDE.

Perea, A. \& Perea, M. (2014). Desarrollo didáctico de la escritura. Revista Internacional de Filología, Comunicación y sus Didácticas, número 36 -37. Universidad de Sevilla. Sevilla - España: CAUCE.

Rosas, L (2012). Incidencia de la dominancia lateral en la disgrafía motriz. Universidad Central del Ecuador. Quito- Ecuador.

Triones, M. \& y Gallardo, J. (s.f). Psicología de la Educación y del Desarrollo en contextos escolares. Educación Psicología pirámide.

Vilatuña, F. \& otros. (2012) Sensación y percepción en la construcción del conocimiento. Sophia, Colección de Filosofía de la Educación. Universidad Politécnica Salesiana Núm.13 (pp.123-149). 